\title{
THE CONSERVATION STATE OF CHARACIDAE FISH (PISCES: CHARACIFORMES) IN AN AREA OF THE PLATA BASIN, ARGENTINA
}

\author{
ESTADO DE CONSERVACION DE PECES CHARACIDAE (PISCES: \\ CHARACIFORMES) EN UN AREA DE LA CUENCA DEL PLATA, ARGENTINA
}

\author{
Marcelo A Zayas \& Elly Cordiviola
}

Instituto Nacional de Limnología (INALI-CONICET-UNL), José Maciá 1933, (3016)

Santo Tomé, Santa Fe (Argentina). E-mails: marcelozayas@ hotmail.com; ellycordi@yahoo.com.ar.

\begin{abstract}
The fish conservation was quantified by means of the index SUMIN, composed by the summation of 12 outstanding variables for the species survival and/or conservation. An arranged list of 53 species was obtained according to a gradient of conservation necessity. The species determined as vulnerable of maximum priority: Salminus brasiliensis, Pygocentrus nattereri, Serrasalmus marginatus and S. rhombeus; species vulnerable of special attention: Brycon orbygnianus, Triportheus paranensis, Serrasalmus serrulatus, S. spilopleura, Piaractus mesopotamicus, Hemigrammus mattei, Cynopotamus argenteus, Astyanax correntinus, Gymnocorymbus ternetzi, Hyphessobrycon anisitsi and Mylossoma orbignyanus. The remaining species, were qualified as not threatened: Poptella paraguayensis, Astyanax abramis, A. bimaculatus, A. fasciatus, A. taeniatus, A. erythropterus, A. lineatus, A. paranahybae, Charax stenopterus, Roeboides bonariensis, R. paranensis, Pseudocorynopoma doriai, Moenkhausia dichroura, M. sanctaefilomenae, Mylossoma paraguayensis, Odontostilbe microcephala, Galeocharax humeralis, Aphyocharax anisitsi, A. rathbuni, A. nasutus, A. paraguayensis, Bryconamericus stramineus, Oligosarcus oligolepis, O. jenynsi, Odontostilbe pequira, Metynnis mola, M. otuquensis, Prionobrama paraguayensis, Bryconamericus iheringi, Hyphessobrycon igneus, $H$. meridionalis, H. eques, H. reticulatus, Tetragonopterus argenteus, Clupeacharax anchoveoides, Markiana nigripinnis, Cheirodon interruptus and Cynopotamus kincaidi. This index allows estimating quantitatively the conservation state of the ichthyic species.
\end{abstract}

Keywords: Conservation, fish, Characidae, South America

\section{RESUMEN}

La conservación de peces se cuantificó mediante la aplicación de un índice (SUMIN), compuesto por la suma de 12 variables relevantes para su supervivencia y/o conservación. Se obtuvo un listado jerarquizado de 53 especies, de acuerdo a un gradiente de necesidad de conservación. Se determinaron como especies vulnerables de máxima prioridad: Salminus brasiliensis, Pygocentrus nattereri, Serrasalmus marginatus y S. rhombeus; especies vulnerables de especial atención: Brycon orbygnianus, Triportheus paranensis, Serrasalmus serrulatus, S. spilopleura, Piaractus mesopotamicus, Hemigrammus mattei, Cynopotamus argenteus, Astyanax correntinus, Gymnocorymbus ternetzi, Hyphessobrycon anisitsi y Mylossoma orbignyanus. Las especies restantes, fueron calificadas como no amenazadas: Poptella paraguayensis, Astyanax abramis, A. bimaculatus, A. fasciatus, A. taeniatus, A. erythropterus, A. lineatus, A. paranahybae, Charax stenopterus, Roeboides bonariensis, $R$. paranensis, Pseudocorynopoma doriai, Moenkhausia dichroura, M. sanctaefilomenae, Mylossoma paraguayensis, Odontostilbe microcephala, Galeocharax humeralis, Aphyocharax anisitsi, A. rathbuni, A. nasutus, A. paraguayensis, Bryconamericus stramineus, Oligosarcus oligolepis, O. jenynsi, Odontostilbe pequira, Metynnis mola, M. otuquensis, Prionobrama paraguayensis, Bryconamericus iheringi, Hyphessobrycon igneus, H. meridionalis, H. eques, H. reticulatus, Tetragonopterus argenteus, Clupeacharax anchoveoides, Markiana nigripinnis, Cheirodon interruptus y Cynopotamus kincaidi. Este índice permite una apreciación cuantitativa de la situación de conservación de las especies ícticas.

Palabras Claves: Conservación, peces, Characidae, Sud America 


\section{INTRODUCTION}

Argentina is one of the countries with more biomass diversity, with all type of climates and physiographic conditions, giving place to the maximum environmental variation. It is zoogeographically located inside the Neotropical Region, conformed by big hydrographical systems as Amazon, Orinoco, Paraná-Plata, etc.

The Argentinian continental ichthyofauna is very well represented, 450 fish species, with the absence of the families Osteoglossidae and Nandidae (López 2001).

There is little knowledge of the ichthyic fauna conservation state. There are no previous records of the Santa Fe Province, Argentina only a listing of species of the family Characidae, (del Barco 1997), with the categorization according to its sport, commercial, eating, ornamental or bait value, and a catalogue (Cazenave \& Bacchetta 2001), with an inventory of the species belonging to the Florentino Ameghino Natural Science Museum of the city of Santa Fe.

Several authors classify the Argentinean ichthyic fauna, having into account different fish groups methodologies (Bertonatti \& González 1993; Chebez, 1994; Orlandini et al. 2001).

The objective is to quantify, with the application of a numerical index, the fish conservation state of the family Characidae in an area of the Plata Basin (Santa Fe Province, Argentina), taking into account the biological, ecological, ethological and distribution variables.

\section{MATERIAL AND METHODS}

Study AREA

It is located inside the Paranoplatensean Ichthyogeographical Province, Paranensean Domain, Brasílic Subregion (Ringuelet 1975), with 147 species of Characiformes (López 2001). According to the ecoregions outlined by López, et al. 2002, it is be located in the Subtropical Potamic Axis Ecoregion.

The area studied of Santa Fe Province $\left(28^{\circ}-34^{\circ} \mathrm{L}\right.$ $\mathrm{S}$ and $59^{\circ}-63^{\circ} \mathrm{L} \mathrm{W}$ ) is geomorphologically an uninterrupted plain, tectonically is part of the pampasia, the basement belong to the Brasilia Massif, fractured and divided into sunken blocks of different depths. The most important faults go from $\mathrm{N}$ to $\mathrm{S}$; one of them is now occupied, by the Paraná River. To the north, it presents a warm climate with an annual mean temperature of $21^{\circ} \mathrm{C}$; annual precipitations among 800 and $1100 \mathrm{~mm}$ that diminish to the west. To the south, it is mild with an annual average of $17^{\circ} \mathrm{C}$ and with an annual precipitation average of $944 \mathrm{~mm}$.

The Santa Fe Province rivers belong to La Plata Basin and they drain directly or indirectly in their main bed: the Paraná River. It is the second most important fluvial course of South America after the Amazon, and due to their uses, constitutes the most important system of South America (del Barco, 2000). The stretch named Middle Paraná develops an large flood valley, integrating a complex fluvial net with low and flooding islands. It is the main productivity support due to a recurrent process of growing and low water (flood pulse, Junk et al. 1989) that allows incorporating to the environments the biomass synthesized in the alluvial plain during the period of low water. It runs from north to south along the east of the Santa Fe Province, being their main tributaries the Salado and Carcarañá Rivers.

Conservation Index

Fifty-three species of fish of the family Characidae mentioned by del Barco 1997, and Cazenave \& Bacchetta 2001 were studied. López et al. (2003) (Table I) was followed for the systematic arrangement.

The method proposed by Bello \& Úbeda (1998), adapted from Reca et al. (1994), was applied considering the index SUMIN, summation of 12 variables, referred to the biology, ecology, ethology, distribution, etc. of each species.

The mean and standard deviation was calculated for the group of species under study. To qualify the conservation state, the species were grouped as follow: those ones whose SUMIN value overcomes the mean plus a standard deviation are considered vulnerables of maximum priority. Those that are between the mean and this one plus a standard deviation, are vulnerables requiring special attention and those that have a similar value to the mean or minor are not threateneds.

THE APPLIED VARIABLES ARE:

1) Continental distribution: area where the species is, it allows understand its geographical contiguity, 
which is associated with the idea of genetic flow among the populations. 2) National distribution: presence in the main hydrographical basins of the country. 3) Amplitude in the habitat use: aptitude to live in different environments. 4) Width in the vertical space use: The portions employed are quantified for feeding, reproduction and breathing. 5) Corporal size: maximum overall length. 6) Reproductive potential: type of fertilization, ovocytes size and parental cares, according to Bello y Ubeda (1998). 7) Trophic width: trophic level. 8) Abundance: size of the populations in the country, by means of relative abundance scales. 9) Taxonomic singularity: presence of unique DNA sequences inside the different populations. 10) Singularity: particular characteristics that may affect its survival and / or conservation. 11) Extractive actions: anthropic activities that imply the individuals removal. 12) Degree of protection: number of actual actions for the species conservation inside the country; the classification carried out by other authors and the Provincial Law 12.212, (2003), related to the fish capture, were considered as such and denominated conservation units.

The necessary information for each species considered was obtained from: Alonso de Arámburu (1953); Freyre et al. (1967); Pignalberi (1967), (1975); Ringuelet et al. (1967), Ringuelet (1975); Bonetto et al. (1969), (1970a), (1970b), (1978), (1981); Destefanis \& Freyre (1972); Menezes (1976); Cordiviola de Yuan \& Oliveros (1979); Cordiviola de Yuan (1980); Oliveros (1980); Miquelarena et al. (1981); Freyre \& Iwaszkiw (1982); Miquelarena \& Casciotta (1982); Beltzer (1983); Escalante (1983); Iwaszkiw et al. (1983); Cordiviola de Yuan et al. (1984); Cordiviola de Yuan \& Pignalberi (1985); Haro \& Gutierrez (1985); Oldani \& Tablado (1985); Freyre \& Mollo (1987); Pignalberi \& Cordiviola de Yuan (1988); Tablado et al. (1988); Rossi (1989); Winemiller \& Kira (1989); Casciotta et al. (1989), (1991); Oldani (1990); del Barco (1990), (1997); Aquino (1991); Almirón et al. (1992); Rossi \& Parma de Croux (1992); Bertonatti \& González (1993); Braga (1993/94), (1994); Chebez (1994); Miquelarena \& Aquino (1995); Liotta et al. (1995/96); Vazzoler (1996); López et al. (1996), (2003); Bello \& Úbeda (1998); de la Peña (1998); Sverlij et al. (1998); Briski et al. (1999); Roa \& Permingeat (1999); Cazenave \& Bacchetta (2001); Nakatami et al. (2001); Orlandini et al. (2001); Drago et al. (2003) and Reis et al. (2003).
TABle I. Family Characidae species registered in an area of La Plata Basin (Santa Fe Province, Argentina) according to del Barco (1997) and Cazenave \& Bachetta (2001). The classification and taxonomic arrangement according to López et al. (2003).

Tabla I. Especies de la familia Characidae registradas en un área de la Cuenca del Plata ( Provincia de Santa $\mathrm{Fe}$, Argentina) según del Barco (1997) y Cazenave \& Bacheta (2001). Para el ordenamiento y actualización taxonómica se siguió a López et al. (2003).

\section{ACTINOPTERYGII \\ NEOPTERYGII \\ CHARACIFORMES \\ CHARACIDAE \\ Characinae}

Charax stenopterus (Cope 1894)

Roeboides bonariensis (Steindachner 1879)

R. paranensis Pignalberi 1975

Cynopotaminae

Cynopotamus argenteus (Valenciennnes 1836)

C. kincaidi (Schultz 1950)

Galeocharax humeralis (Valenciennes 1847)

Bryconinae

Brycon orbygnianus (Valenciennes 1849)

Salmininae

Salminus brasiliensis Cuvier 1816

Triportheinae

Triportheus paranensis (Günther 1874)

Clupeacharacinae

Clupeacharax anchoveoides Pearson 1924

Paragoniatinae

Prionobrama paraguayensis (Eigenmann 1914)

Aphyocharacinae

Aphyocharax anisitsi Eigenmann \& Kennedy 1903

A. nasutus Ahl, 1936

A. paraguayensis (Eigenmann 1915)

A. rathbuni Eigenmann 1907

Glandulocaudinae

Pseudocorynopoma doriai Perugia 1891

Stethaprioninae

Poptella paraguayensis (Eigenmann 1907)

Tetragonopterinae

Astyanax abramis (Jenyns 1842)

A. bimaculatus (Linné 1758)

A. correntinus (Holmberg 1891)

A. erythropterus (Holmberg 1891)

A. fasciatus (Cuvier 1819)

A. lineatus (Perugia 1891)

A. paranahybae Eigenmann 1911

A. taeniatus (Jenyns 1842)

Bryconamericus iheringi (Boulenger 1887)

B. stramineus Eigenmann 1908

Gymnocorymbus ternetzi (Boulenger 1895) 
Conservation of Characidae fish: ZaYAS, M. \& E. Cordiviola

Hemigrammus mattei Eigenmann 1908

Hyphessobrycon anisitsi (Eigenmann 1907)

H. eques (Steindachner 1882)

H. igneus Miquelarena, Menni, López \& Casciotta 1981

H. meridionalis Ringuelet, Miquelarena \& Menni 1978

H. reticulatus Ellis 1911

Markiana nigripinnis (Perugia 1891)

Moenkhausia dichroura (Kner 1858)

M. sanctaefilomenae (Steindachner 1907)

Tetragonopterus argenteus Cuvier 1817

Oligosarcus oligolepis (Steindachner 1867)

O. jenynsi (Günther 1864)

Cheirodontinae

Cheirodon interruptus (Jenyns 1842)

Odontostilbe microcephala Eigenmann 1907

O. pequira (Steindachner 1882)

Serrasalminae

Pygocentrus nattereri Kner 1858

Serrasalmus marginatus Valenciennes 1836

S. rhombeus Linné 1766

S. serrulatus (Valenciennes 1850)

S. spilopleura Kner 1858

Myleinae

Metynnis mola Eigenmann y Kennedy 1903

M. otuquensis Ahl 1923

Mylossoma orbignyanus (Valenciennes 1850)

M. paraguayensis Norman 1929

Piaractus mesopotamicus (Holmberg 1891)

\section{RESULTS}

The species were ordered decreasingly according to the SUMIN value (Table II). The SUMIN mean for the Characidae consireded $=7 \pm 2$ (between 3 and 12)

The vulnerable species of maximum priority are: Salminus brasiliensis, Pygocentrus natteri, Serrasalmus marginatus and $S$. rhombeus. The first one presented the maximum of the SUMIN (12) due to its corporal size, it is one of the larger species within the family, until $77 \mathrm{~cm}$. (Ringuelet et al. 1967 and Almirón et al. 1992) and extractive actions, due to its commercialization and sport fishing. The Provincial Law 12.212, (2003), that settles down the period, form and size of the allowed captures. The remaining ones are some of the called "palometas" or "piranhas", with medium sizes and extractive activities of smaller impact, due to the social repulsion generated by their aggressiveness.
TABLE II. Arranged list of Characidae species of an area of La Plata Basin (Santa Fe Province, Argentina), according to the SUMIN conservation index. The state of conservation is indicated.

TABla II. Lista ordenada de especies de Characidae de un área de la Cuenca del Plata (Provincia de Santa Fe, Argentina), de acuerdo al índice de conservación SUMIN. Se indica el estado de conservación

\begin{tabular}{|c|c|}
\hline Species & SUMIN \\
\hline \multicolumn{2}{|c|}{ Vulnerables of maximun priority } \\
\hline Salminus brasiliensis & 12 \\
\hline Pygocentrus nattereri & 10 \\
\hline Serrasalmus marginatus & 10 \\
\hline Serrasalmus rhombeus & 10 \\
\hline \multicolumn{2}{|l|}{ Vulnerables of special attention } \\
\hline Brycon orbygnianus & 9 \\
\hline Triportheus paranensis & 9 \\
\hline Serrasalmus serrulatus & 9 \\
\hline Serrasalmus spilopleura & 9 \\
\hline Piaractus mesopotamicus & 9 \\
\hline Hemigrammus mattei & 8 \\
\hline Cynopotamus argenteus & 8 \\
\hline Astyanax correntinus & 8 \\
\hline Gymnocorymbus ternetzi & 8 \\
\hline Hyphessobrycon anisitsi & 8 \\
\hline Mylossoma orbignyanus & 8 \\
\hline \multicolumn{2}{|l|}{ No threatened } \\
\hline Poptella paraguayensis & 7 \\
\hline Astyanax taeniatus & 7 \\
\hline Charax stenopterus & 7 \\
\hline Roeboides bonariensis & 7 \\
\hline Roeboides paranensis & 7 \\
\hline Pseudocorynopoma doriai & 7 \\
\hline Astyanax erythropterus & 7 \\
\hline Moenkhausia dichroura & 7 \\
\hline Mylossoma paraguayensis & 7 \\
\hline Hyphessobrycon reticulatus & 6 \\
\hline Odontostilbe microcephala & 6 \\
\hline Galeocharax humeralis & 6 \\
\hline Aphyocharax anisitsi & 6 \\
\hline Aphyocharax nasutus & 6 \\
\hline Aphyocharax paraguayensis & 6 \\
\hline Astyanax lineatus & 6 \\
\hline Astyanax paranahybae & 6 \\
\hline Bryconamericus stramineus & 6 \\
\hline
\end{tabular}




\begin{tabular}{lc}
\hline Species & SUMIN \\
\hline Hyphessobrycon eques & 6 \\
Oligosarcus oligolepis & 6 \\
Odontostilbe pequira & 6 \\
Metynnis mola & 6 \\
Metynnis otuquensis & 6 \\
Oligosarcus jenynsi & 6 \\
Prionobrama paraguayensis & 5 \\
Astyanax abramis & 5 \\
Astyanax bimaculatus & 5 \\
Bryconamericus iheringi & 5 \\
Hyphessobrycon igneus & 5 \\
Hyphessobrycon meridionalis & 5 \\
Moenkhausia sanctaefilomenae & 5 \\
Tetragonopterus argenteus & 5 \\
Clupeacharax anchoveoides & 5 \\
Astyanax fasciatus & 5 \\
Markiana nigripinnis & 4 \\
Cheirodon interruptus & 4 \\
Aphyocharax rathbuni & 4 \\
Cynopotamus kincaidi & 3 \\
\hline
\end{tabular}

Vulnerable species of special attention are: Brycon orbygnianus, Cynopotamus argenteus, Triportheus paranensis, Astyanax correntinus, Gymnocorymbus ternetzi, Hemigrammus mattei, Hyphessobrycon anisitsi, Serrasalmus serrulatus, S. spilopleura, Mylossoma orbignyanus and Piaractus mesopotamicus. Most of them are present all through South America and only three are continuous in less than half of this continent. Astyanax correntinus, Hemigrammus mattei, and Mylossoma orbignyanus inhabit only the La Plata Basin. There are small species, as well as other few medians, as Brycon orbignyanus, Serrasalmus spilopleura and Cynopotamus argenteus, and, exceptionally, a great size species, Piaractus mesopotamicus ("pacú”) $82 \mathrm{~cm}$ (Ringuelet et al. 1967). In this group, no information was verified for the reproduction variable. They are mainly carnivorous species, either generalists or specialists. Few of them are protected by the conservation units: Gymnocorymbus ternetzi (commercially threatened, Bertonatti \& González, 1993) and Mylossoma orbygnianus (uncertain,
Chebez, 1994, not known, Bertonatti \& González, 1993). The Provincial Law 12.212, (2003), protects Brycon orbignyanus and Piaractus mesopotamicus. Some are caught by sport fishing, for the aquariums or consumption.

No threatened species are: A styanax abramis, $A$. bimaculatus, A. fasciatus, A. lineatus, A. paranahybae, A. taeniatus, A. erythropterus, Aphyocharax anisitsi, A. nasutus, $A$. paraguayensis, A. rathbuni, Charax stenopterus, Roeboides bonariensis, $R$. paranensis, Pseudocorynopoma doriai, Moenkhausia dichroura, M. sanctaefilomenae, Mylossoma paraguayensis, Odontostilbe microcephala, $O$. pequira, Galeocharax humeralis, Oligosarcus oligolepis, O. jenysi, Metynnis mola, M. otuquensis, Prionobrama paraguayensis, Bryconamericus iheringi, B. stramineus, Hyphessobrycon reticulatus, $H$. igneus, $H$. meridionalis, $H$. eques, Tetragonopterus argenteus, Markiana nigripinnis, Cheirodon interruptus, Poptella paraguayensis, Clupeacharax anchoveoides, and Cynopotamus kincaidi. Ninety-five per cent (36 out of 39 spp.) are distributed all along South America or in most of it, and Astyanax erythropterus, and Hyphessobrycon igneus are only in less than half of this continent, in continuous form.

All the species are small, measuring less than 25 $\mathrm{cm}$ of total length. Regarding the reproduction, Oligosarcus jenynsi, Astyanax bimaculatus, A. fasciatus, Bryconamericus iheringi and Tetragonopterus argenteus, lay numerous eggs, and do not present parental cares. They are protected by two or more conservation units: Cheirodon interruptus, Aphyocharax rathbuni, Cynopotamus kincaidi, Mylossoma paraguayensis, Metynnis mola, M. otuquensis, Bryconamericus iheringi, Hyphessobrycon meridionalis, H. igneus, Clupeacharax anchoveoides, and Markiana nigripinnis; for one or more conservation units: Aphyochatrax anisitsi, Oligosarcus jenynsi, Prionobrama paraguayensis, Astyanax fasciatus, Pseudocorynopoma doriai, and Hyphessobrycon eques; the other ones are not protected by any of the conservation units considered in this analysis: Charax stenopterus, Roeboides bonariensis, $R$. paranensis, Aphyocharax paraguayensis, $A$. nasutus, Monkhausia dichroura, $M$. sanctafilomenae, Galeocharax humeralis, 
Oligosarcus oligolepis, Astyanax abramis, A. bimaculatus, A. lineatus, A. paranahybae, A. taeniatus, A. erythropterus, Tetragonopterus argenteus, Hypessobrycon reticulatus, Odontostilbe microcephala, O. pequira, Bryconamericus stramineus, and Poptellaparaguayensis.

\section{DISCUSSION AND CONCLUSIONS}

Conservation works mention only 21 of the 53 species considered (Table III). Bertonatti \& González (1993) and Chebez (1994), apply similar methodologies for obtaining the information and at the level of certainty demanded to ponder the criteria. They use the qualification proposed by the
World Union for the Nature UICN (1990). The first ones provide a list of species, assigning categories of conservation, by means of a qualitative appreciation and a global interpretation of the situation of each species, without giving precise foundations.

Chebez (1994) highlights, in the uncertain and strange species, the lack of information concerning its distribution, what coincides with the conclusions of the present work.

The methodology applied, adapted to fish, is a useful tool for the evaluation of the ichthyic resource conservation, although in some of the considered species, the lack of information on reproduction and food habitats hindered obtaining an adjusted valuation.

TABLE III. Comparison of the results obtained applying the SUMIN in fish Characidae of an area of la Plata Basin (Santa Fe Province, Argentina), with those obtained by other authors. U= uncertain; IK = insufficiently known; S= strange; $\mathrm{CT}=$ commercially threatened; $\mathrm{VMP}=$ vulnerable of maximum priority; $\mathrm{VSA}=$ vulnerable of special attention; $\mathrm{NT}=$ no threatened; $\mathrm{w} / \mathrm{d}=$ without data.

TABLA III. Comparación de los resultados obtenidos aplicando el SUMIN para peces Characidae de un área de la Cuenca del Plata (Provincia de Santa Fe, Argentina), con los obtenidos por otros autores. U= Indeterminada; IK= Insuficientemente Conocida; $\mathrm{S}=\mathrm{Rara}$; $\mathrm{CT}=$ Comercialmente Amenazada; VMP=Vulnerable de Máxima Prioridad; $\mathrm{VSA}=$ Vulnerable que merece Especial Atención; NT= No Amenazada; w/d= sin datos.

\begin{tabular}{|c|c|c|c|c|}
\hline Species & SUMIN & Chebez (1994) & $\begin{array}{c}\text { Bertonatti y } \\
\text { González (1993) }\end{array}$ & $\begin{array}{l}\text { Orlandini et } \\
\text { al. (2001) }\end{array}$ \\
\hline Salminus brasiliensis & VMP (12) & $\mathrm{w} / \mathrm{d}$ & IK & $\mathrm{w} / \mathrm{d}$ \\
\hline Piaractus mesopotamicus & VSA (9) & $\mathrm{w} / \mathrm{d}$ & $\mathrm{w} / \mathrm{d}$ & $\mathrm{w} / \mathrm{d}$ \\
\hline Brycon orbygnianus & VSA (9) & $\mathrm{U}$ & IK & w/d \\
\hline Mylossoma orbignyanus & VSA (8) & $\mathrm{U}$ & IK & $\mathrm{w} / \mathrm{d}$ \\
\hline Gymnocorymbus ternetzi & VSA (8) & $\mathrm{w} / \mathrm{d}$ & $\mathrm{CT}$ & $\mathrm{w} / \mathrm{d}$ \\
\hline Pseudocorynopoma doriai & NT (7) & $\mathrm{w} / \mathrm{d}$ & $\mathrm{CT}$ & $\mathrm{w} / \mathrm{d}$ \\
\hline Mylossoma paraguayensis & NT (7) & $\mathrm{U}$ & IK & w/d \\
\hline Oligosarcus jenynsi & NT (6) & $\mathrm{w} / \mathrm{d}$ & $\mathrm{w} / \mathrm{d}$ & NT \\
\hline Metynnis otuquensis & NT (6) & $\mathrm{U}$ & IK & w/d \\
\hline Metynnis mola & NT (6) & $\mathrm{U}$ & IK & $\mathrm{w} / \mathrm{d}$ \\
\hline Hyphessobrycon eques & NT (6) & $\mathrm{w} / \mathrm{d}$ & $\mathrm{CT}$ & $\mathrm{w} / \mathrm{d}$ \\
\hline Aphyocharax anisitsi & NT (6) & $\mathrm{w} / \mathrm{d}$ & $\mathrm{CT}$ & w/d \\
\hline Prionobrama paraguayensis & NT (5) & $\mathrm{w} / \mathrm{d}$ & $\mathrm{CT}$ & $\mathrm{w} / \mathrm{d}$ \\
\hline Hyphessobrycon meridionalis & NT (5) & $\mathrm{S}$ & $\mathrm{S}$ & $\mathrm{w} / \mathrm{d}$ \\
\hline Hyphessobrycon igneus & NT (5) & S & $S$ & $\mathrm{w} / \mathrm{d}$ \\
\hline Clupeacharax anchoveoides & NT (5) & S & S & w/d \\
\hline Bryconamericus iheringi & NT (5) & $S$ & $S$ & $\mathrm{w} / \mathrm{d}$ \\
\hline Astyanax bimaculatus & NT (5) & w/d & w/d & NT \\
\hline Markiana nigripinnis & NT (4) & $\mathrm{U}$ & IK & $\mathrm{w} / \mathrm{d}$ \\
\hline Cheirodon interruptus & NT (4) & S & S & NT \\
\hline Aphyocharax rathbuni & NT (4) & $S$ & $S$ & $\mathrm{w} / \mathrm{d}$ \\
\hline Cynopotamus kincaidi & NT (3) & $\mathrm{U}$ & IK & w/d \\
\hline
\end{tabular}




\section{BIBLIOGRAPHY}

Almirón, A.E., S.E. Gómez \& N.I. Toresani. 1992. Peces de agua dulce de la provincia de Buenos Aires, Argentina. Comisión de Investigaciones Científicas provincia de Buenos Aires. 2 (12): 129.

Alonso de Aramburu, A. 1953. Contribución a la sistemática de los peces carácidos llamados "Dientudos". Notas Museo de La Plata. 16 (Zool. 145): 297-320.

Aquino, A.E. 1991. Alimentación de Oligosarcus jenynsii (Günther 1864) (Osteichthyes, Characidae) en el embalse El Cadillal (Tucumán, Argentina). Biología Acuática. 15 (2): 176-177.

Bello, M.T. \& C.A. UbedA. 1998. Estado de conservación de los peces de agua dulce de la Patagonia Argentina. Aplicación de una metodología objetiva. Gayana Zoológica. 62 (1): 45-60.

BeLtzer, A.H. 1983. Nota sobre fidelidad y participación trófica del "Biguá común" (Phalacrocorax olivaceus) en ambientes del río Paraná Medio (Pelecaniformes: Phalacrocoracidae). Revista de la Asociación de Ciencias Naturales del Litoral. 14 (2): $111-114$

Bertonatti, C. \& F. González. 1993. Lista de vertebrados argentinos en peligro de extinción. Boletín Técnico Fundación Vida Silvestre Argentina. 8: 1-39.

Bonetto, A.A., E. Cordiviola de Yuan, C. Pignalberi \& O. Oliveros. 1969. Ciclos hidrológicos del río Paraná y las poblaciones de peces contenidas en las cuencas temporarias de su valle de inundación. Physis 29 (78): 213-223.

Bonetto, A.A., E. Cordiviola de Yuan \& C. Pignalberi. 1970a. Nuevos datos sobre poblaciones de peces en ambientes leníticos permanentes del Paraná medio. Physis 30 (80): 141-154.

Bonetto, A.A., C. Pignalberi, E. Cordiviola de Yuan \& O. Oliveros. 1970b. Nuevos aportes al conocimiento de las poblaciones ícticas en cuencas temporarias del valle de inundación del Paraná medio. Acta Zoologica Lilloana 27: 135153

Bonetto, A.A., D. Roldán \& M. Esteban Oliver. 1978. Estudios limnológicos en la cuenca del Riachuelo (Corrientes, Argentina). I Poblaciones de peces en ambientes leníticos y lóticos. Ecosur 5 (9): 115.

Bonetto, A.A., D. Roldán \& M. Canon Verón. 1981. Algunos aspectos estructurales y ecológicos de la ictiofauna del sistema del Iberá (Corrientes, Argentina). Ecosur 8 (15): 79-89.

Braga, L. 1993/94. Las especies del género Metynnis (Teleostei, Characiformes) en Argentina. Revista de Ictiología 2/3 (1/2): 27-34.

Braga, L. 1994. Los Characidae de Argentina de las subfamilias Cynopotaminae y Acestrorhynchinae. Fauna de Agua Dulce de la República Argentina 40 (6): 45p.
Britski, H.A., K.Z. de S. de Silimon \& B.S. Lopes. 1999 Peixes do Pantanal. Manual de identificaçao. Empresa Brasileira de Pesquisa Agropecuaria. Serviço de Produçao de Informaçao 184 pp.

Casciotta, J.R., H.L. Lopez, R.C. Menni \& A.M. Miquelerena. 1989. The first faune from the Salado River (Central Argentina, South América) with additions to the Dulce River and linnológical comments. Archiv für Hydrobiologie 115 (4): 603612 .

Casciotta, J.R., A.M. Miquelarena \& L.C. Protogino. 1991. Una especie de Odontostilbe (Teleostei, Characidae) para la cuenca del río Uruguay, con comentarios sobre los caracteres diagnósticos del género. Biología Acuática 15 (2): 164-165.

Cazenave, J. \& C. Bacchetta. 2001. Catálogo de peces argentinos de agua dulce del Museo Provincial de Ciencias Naturales "Florentino Ameghino". Comunicación Provincial del Museo de Ciencias Naturales "Florentino Ameghino" 18 pp.

Chebez, J.C. 1994. Los que se van. Especies argentinas en peligro. Editorial. Albatros, Buenos Aires 604 pp.

Cordiviola de Yuan, E. 1980. Campaña limnológica "Keratella I"en el río Paraná Medio: Taxocenos de peces de ambientes leníticos. Ecología 4: 103113.

Cordiviola de Yuan, E., N. Oldani, O. Oliveros \& C. Pignalberi de Hassan. 1984. Aspectos limnológicos de ambientes próximos a la ciudad de Santa Fe (Paraná Medio): poblaciones de peces ligadas a la vegetación. Neotrópica 30 (84): 127 139.

Cordiviolade Yuan, E. \& O.B. Oliveros. 1979. Campaña "Keratella I" a lo largo del río Paraná medio I. Peces de ambientes leníticos. Acta Zoológica Lilloana 35: 629-642.

Cordiviola de Yuan, E \& C. Pignalberi. 1985. Fish populations in the Paraná River: Lenitic environments of Diamante and San Pedro areas. (Argentine Republic). Hydrobiologia 127: 213218.

De La PeÑa, M. 1998. Guía de peces, anfibios, reptiles y mamíferos del Litoral Argentino. Fundación Habitat \& Desarrollo 367 pp.

Del B arco, D.M. 1990. Alimentación de Salminus maxillosus Valenciennes 1810, en distintos ambientes de la provincia de Santa Fe (Pisces, Characidae). Revista de la Asociación de Ciencias Naturales del Litoral 21(2): 159-166.

Del Barco, D.M. 1997. Lista de peces de la provincia de Santa Fe. Anexo I; 97-106. In: Rozzati JC \& E Mosso (Eds.). Sistema provincial de áreas naturales protegidas de Santa Fe. Gobierno de la Provincia de Santa Fe, Administración de Parques Nacionales, Edición de la Asociación Cooperadora de la Estación Zoológica Experimental de Santa Fe, Argentina, 174 pp.

Del Barco, D.M. 2000. Desarrollo sustentable de los recursos pesqueros en aguas continentales. 
Seminario Internacional de Pesca Continental. Consejo Federal de Inversiones (CFI) 13 pp.

Destefanis, S. \& L. Freyre. 1972. Relaciones tróficas de los peces de la Laguna de Chascomús con un intento de referenciacíon ecológica y tratamiento bioestadístico del espectro trófico. Acta Zoológica Lilloana 29: 17-33.

Drago, E.C., I.E. De Drago, O.B. Oliveros \&A.R. Paira. 2003. Aquatic habitats, fish and invertebrate assemblages of the Middle Paraná River. Amazoniana 17 (3/4): 291-341.

Escalante, A.H. 1983. Contribución al conocimiento de las relaciones tróficas de peces de agua dulce del área platense. II. Otros Tetragonopteridae. Limnobios 2: 379-402.

Freyre, L.R. \& J. Iwaszkiw. 1982. Importancia de la distribución espacial de ovocitos en ovarios de peces para determinación de fecundidad. Limnobios 2 (5): 305-310.

Freyre, L., R. I riart, R.A. Ringuelet, C. Togo \& J. Z etti. 1967. Primeros resultados sobre estimaciones de poblaciones de peces de lagunas pampásicas. Physis 26 (73): 421-433.

Freyre, L.R. \& S.M. Mollo. 1987. Estudio biométrico para estimar el tamaño y peso de los peces a partir de las dimensiones de sus otolitos. Revista de la Asociación de Ciencias Naturales del Litoral 18 (2): 145-154.

Haro, J.G. \& M. Gutiérrez. 1985. Alimentación de Oligosarcus jenynsii (Günther) (Pisces, Characidae) en el lago San Roque (Córdoba, Argentina). Revista de la Asociación de Ciencias Naturales del Litoral 16 (2): 227-235.

IwASZKIw, J.M., L.R. F REYRE \& E.D. SENDRA. 1983. Estudio de la maduración, época de desove y fecundidad del dientudo Oligosarcus jenynsii (Pisces Characidae) del Embalse Río Tercero, Córdoba, Argentina. Limnobios 2 (7): 518-525.

Junk, W.K., B ayley P.B. \& R.A. Sparks. 1989. The flood pulse concept in river-floodplain systems. In: Dodge DP (ed.) Proceedings of the International Large River Symposium. Canadian Special Publication Fisheries Aquatic Sciences 106: 110127.

LEY PROVINCIAL DE SANTA FE N 12.212 . Cámara de Senadores de la Provincia de Santa $\mathrm{Fe}$ (Argentina). 20 de noviembre de 2003.

Liotta, J., B. Giacosa \& M. Wagner. 1995/96. Lista comentada de la ictiofauna del delta del río Paraná. Revista de Ictiología 4 (1-2): 23-32.

LÓPEZ, H.L. 2001. Estudio y uso sustentable de la biota austral: Ictiofauna Continental Argentina. Resúmenes del II Taller Internacional de Contaminación y Protección del Medio Ambiente, La Habana, Cuba. Revista Cubana de Investigación Pesquera, Suplemento Especial, versión electrónica $39 \mathrm{pp}$.

López, H.L., L.C. Protogino \& A.E. Aquino. 1996. Ictiología Continental de la Argentina: Santiago del Estero, Catamarca, Córdoba, San Luis, La Pampa y Buenos Aires. AQUATEC 3: 1-14.

López, H.C., C. Morgan \& M.J. Montenegro. 2002. Icthyological ecoregions of Argentina. Probiota, Serie Documentos $\mathrm{N}^{\circ}$ 1: $69 \mathrm{pp}$.

López, H.L., A.M. M iquelarena \& R. Menni. 2003. Lista comentada de los peces continentales de la Argentina. ProBiota Serie Técnica y Didáctica 5: $86 \mathrm{pp}$.

Menezes, N.A. 1976. On the Cynopotaminae, a new subfamily of Characidae (Osteichthyes, Ostariophysi, Characoidei) Arquivos de Zoologia 28 (2): 1-91.

Miquelarena, A.M. \& A.E. Aquino. 1995. Situación taxonómica y geográfica de Bryconamericus thomasi Fowler, 1940 (Teleostei, Characidae) Revista Brasilera de Biologia 55 (4): 559-569.

Miquelarena, A.M., R.H. Arámburu, R.C. Menni \& H. LÓPEZ. 1981. Nuevas localidades para peces de agua dulce de la República Argentina II. Limnobios 2 (2): 127-135.

Miquelarena, A.M. \& J.R. Casciotta. 1982. Presencia en la Argentina de Clupeacharax anchoveoides Pearson, 1924. Limnobios 2 (5): 333-335.

Nakatami, K., A.A. A gostinho, G. Baumgartner, A. Bialetzki, P.V. Sánchez, M.C. Makrakis \& C.S. Pavanelli. 2001. Ovos e larvas de peixes de agua doce. Desenvolvimiento e manual de identificaçao. Editora da Universidade Estadual de Maringá 378 pp.

OldANI, N.O. 1990. Variaciones de abundancia de peces del valle del río Paraná (Argentina). Revista de Hydrobiología Tropical 23 (1): 67-76.

Oldani, N.O. \& A. Tablado. 1985. Dinámica temporal de pequeños peces de agua libre en la laguna "La Cuarentena" (isla Carabajal, río Paraná medio). Studies on Neotropical Fauna and Environment 20 (1): 49-58.

Oliveros, O.B. 1980. Campaña limnológica "Keratella I" en el río Paraná Medio: aspectos tróficos de los peces de ambientes leníticos. Ecología 4: 115126.

Orlandini, S.I., L. Aun \& R. Martori. 2001. Estado de conservación de la ictiofauna de las sierras pampeanas de la provincia de Córdoba, Argentina. Boletín Sociedad Biológica de Concepción 72: 91-103.

Pignalberi, C. 1967. Observaciones sobre gónadas de algunas especies de peces del Paraná medio. Acta Zoológica Lilloana 23: 163-172.

Pignalberi, C. 1975. Una nueva especie del género Roeboides Günther del río Paraná: Roeboides paranensis sp. nov. (Pisces, Characidae). Physis B 34 (89): 151-155.

Pignalberi, C. \& E. Cordiviola de Yuan. 1988. Fish Populations in the Paraguay River waters of the Formosa area, Argentina. Studies on Neotropical Fauna and Environment 23 (3): 165-175.

Reca, A., C. Úbeda \& D. Grigera. 1994. Conservación de la fauna de tetrápodos. I. Un índice para su 
evaluación. Mastozoología Neotropical 1(1): 1728.

Reis, R.E., Kullander S.O. \& C. J. Ferraris. 2003. The check list of the freshwater fishes of South and Central America. Editorial de la Pontificia Universidad Católica de Rio Grande Do Sul $184 \mathrm{pp}$.

Ringuelet, R.A., R.H. Arámburu \& A. Alonso de ArÁmburu. 1967. Los peces argentinos de agua dulce. Comisión Investigaciones Científicas de la Provincia de Buenos Aires, La Plata 602 pp.

Ringuelet, R.A. 1975. Zoogeografía y ecología de los peces de aguas continentales de la Argentina y consideraciones sobre las áreas ictiológicas de América del Sur. Ecosur 2(3): 1-122.

Roa, B.H \& E.D. Permingeat. 1999. Composición y abundancia de la fauna íctica en dos estaciones de muestreo del embalse Yacyretá, Argentina. Revista de Ictiología 7: 49-57.

Rossi, L.M. 1989. Alimentación de larvas de Salminus maxillosus Val. 1840 (Pisces, Characidae). Iheringia Serie Zoológica (69): 49-59.
Rossi, L.M \& M.J. Parma De Croux. 1992. Influencia de la vegetación acuática en la distribución de peces del río Paraná, Argentina. Ambiente Subtropical 2: $65-75$.

Sverlij, S.B., H.L. López, R.L. Delfino Sckenke \& A. Espinach Ros. 1998. Peces del Río Uruguay. Comisión Administradora Río Uruguay 89 pp.

Tablado, A., N.O. Oldani, L. Ulibarrie \& C. Pignalber De Hassan. 1988. Cambios estacionales de la densidad de peces en una laguna del valle aluvial del río Paraná (Argentina). Revista de Hidrobiología Tropical 21 (4): 335-348.

UICN. 2001. Categorías y Criterios de la Lista Roja de la UICN: Versión 3.1. Comisión de Supervivencia de Especies de la UICN. UICN, Gland, Suiza y Cambridge, Reino Unido. ii +33 pp.

VAzzoler, A. 1996. Biología da reproduçao de peixes teleósteos: Teoria e prática. Editora da Universidade Estadual de Maringá 169 pp.

Winemiller, \& O. KIRA. 1989. Patterns of variation in life history among South American fishes in seasonal environments. Oecologia 81: 225-241. 